\title{
全球陆地年降水场的长期变化(1948～2000 年)
}

\author{
施能 陈绿文 \\ (南京气象学院大气科学系, 南京 210044. E-mail: shin@ publicl.ptt.js.cn)
}

\begin{abstract}
摘要 研究了 1948 2000 年全球陆地年降水场的长期变化, 结果表明在 1978 年前后全球年降水量突变 减少, 平均每年减少 $0.54 \mathrm{~mm}$. 模糊聚类方法将 36 个纬圈的年平均降水量划分为 6 个纬带, 研究了 6 个 纬带的年降水量的长期变化. 指出, 除了北半球高纬度 $\left(60^{\circ} \sim 90^{\circ} \mathrm{N}\right)$ 降水略微增加以外, 各纬带的平均降 水量都表现为减少, $35^{\circ} \mathrm{S} 35^{\circ} \mathrm{N}$ 的降水量减少最为显著. 降水量减少最多的地区是热带, 平均降水减少 为 $0.98 \mathrm{~mm} / \mathrm{a}$. 划分了 1948 2000年的全球旱涝年, 指出旱涝年有明显的年代际变化. 研究指出, 在暖事 件全球陆地平均降水量每年减少 $15.4 \mathrm{~mm}$, 而在冷事件年则增加 $14.4 \mathrm{~mm} .20$ 世纪 70 年代末开始频繁发 生的 ENSO 事件可能是全球年降水量减少的一个重要的原因.
\end{abstract}

\section{关键词 全球降水 长期变化 ENSO}

与全球温度相比, 我们对全球降水变化的研究 太少了. 虽然已经有了较多全球降水变化的研究, 例 如 Gruza 等人 ${ }^{[1]}$, Diaz 等人 ${ }^{[2]}$, Bradley 等人 ${ }^{[3]}$, Vinnikov 等人 ${ }^{[4]}$ 和 Hulme ${ }^{[5]}$. 但是, 他们只研究了 20 世纪 80 年代以前的全球降水, 或者 90 年代初的全球 降水 ${ }^{[6-8]}$. 最近, 有些研究涉及 1997 年前后的降水量 资料, 但都是区域降水. Hulme ${ }^{[7]}$ 认为我们还不知道 我们的地球在未来是变干还是变湿. 全球降水变化 问题很需要用最新的资料进行研究.

一般说来, ENSO 年事件与热带大陆的干旱相联 系. Ropelewsi 等人 ${ }^{[9]}$, Lau 等人 ${ }^{[10]}$, Rasmusson 等人 ${ }^{[11]}$ 和 Bradley 等人 ${ }^{[12]}$, 都研究了 ENSO 与全球大尺度降 水问题. 但是上述的研究没有包括 20 世纪 80 年代以 后的 ENSO 事件. 在 Ropelewsi 等人的研究中, 还 不适当地排除了 1982 1983 年的强 ENSO 事件. Wang ${ }^{[13]}$ 指出, 1977 年开始 ENSO 发生了年代际变化, 1977 年以后, ENSO 强而频率高. 1997 1998 年又发生 了近百年最强的 ENSO 事件. 全球年降水与 ENSO 的关系也需要更长的降水资料研究.

\section{1 资料}

本文使用 Chen 等人 ${ }^{[14]}$ 年最新创建的 1948 2000 年全球陆地月降水资料 (PREC/L). PREC/L 资料是 美国 GHCN 最新版本的 17000 个气象站的雨量观测 资料和 CPC 的气候异常监观系统资料相结合的产品. 空间的分辨率是 $2.5^{\circ}\left(-88.75^{\circ} \mathrm{S} \sim 88.75^{\circ} \mathrm{N}\right)$. 全球有 资料的陆地格点数为 4390 , 有严格的检测与质量控 制. Chen 等人 ${ }^{[14]}$ 已经阐明了该资料的优良特性.

\section{2 大尺度年降水场的长期变化}

年降水量的第 1 特征向量图(图略)解释了 $97.2 \%$ 的总方差, 它与 1948 2000年的年降水量的平均图几 乎完全一致, 相似系数高达 0.998. 图 1 是它的时间 权重, 它有明显的下降趋势, 趋势系数为 $-0.344^{[15,16]}$, 达到 0.01 的信度. 图 1 中的虚曲线基本上反映了降水 量的年代际变化. 可以看到, 20 世纪 50 年代是全球 涝期, 这与 Bradley ${ }^{[3]}$ 研究的结果相吻合. 20 世纪 70 年代初中期, 全球降水也比较多, 随后全球降水量明 显减少, 20 世纪 90 年代初至 90 年代中期, 全球降水 是非常少的.

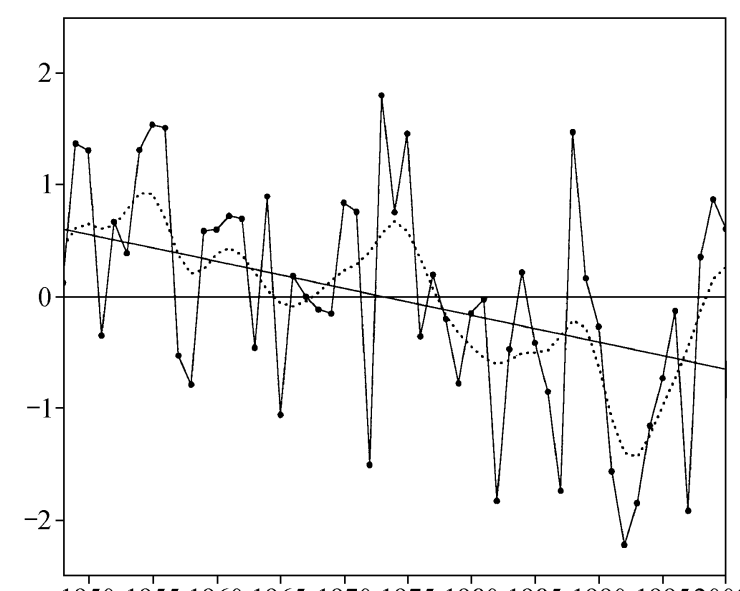

19501955196019651970197519801985199019952000 (年)

图 1 全球年降水量第 1 特征向量的时间系数 (数值已经标准化) 曲线为高斯滤波, 直线为回归方程 


\section{3 年降水量平均距平的长期变化}

我们对全球陆地年降水量计算平均距平, 它的时 间变化(图 2)与图 1 是非常相似的, 相关系数高达 0.82 , 可以看出, 比较明显的突变下降发生在 1978 年左右, 达到 0.01 的显著性. 为进一步证实全球降水量的年 代际变化，制作了 1978 2000 年与 1948 1977 年两时 段的年平均降水量的差值图(图略). 结果表明, 在 4390 个有记录的格点中, 有 3000 个格点的降水量差为 负值, 其中有 1210 个点达到 0.05 以上的统计显著性, 表明全球大范围降水量的减少. 对这个结果我们还进 行了蒙特卡罗模拟检验 ${ }^{[17]}$, 结果表明通过了 0.01 信度. 这样我们认为, 20 世纪 70 年代后期开始, 全球陆地大 尺度降水量突变并明显减少. 对平均距平计算了回归 方程，说明 1948 2000 年全球陆地年降水量平均每年 减少 $0.54 \mathrm{~mm}$, 这个数值是全球 4390 个格点的平均减

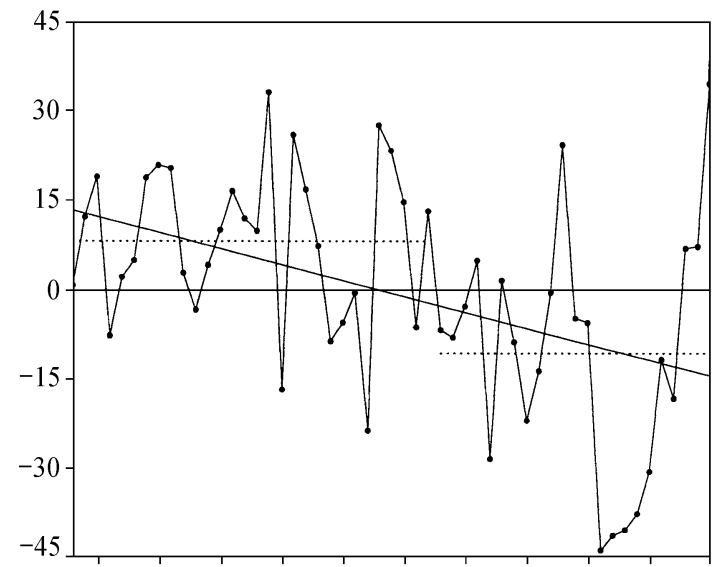

19501955196019651970197519801985199019952000 (年)

图 2 全球平均的年降水量距平的时间变化 (图中数值已标准化)

直线为回归方程, 虚线为两不同时段的平均值
少量. Hulme ${ }^{[5]}$ 指出, 1950 1980 年全球平均降水减少为 $0.4 \mathrm{~mm} / \mathrm{a}$. 本文延长 20 年资料后的值比 Hulme 的值大 $25 \%$, 表示 20 世纪 80 年代以后全球变早更明显了.

根据图 1 和 2 的数值划分全球的旱涝年, 我们认 为这两个序列显示相同特征的年份确定为旱涝年是 比较合适的. 这样, 得到 1948 2000 年全球的旱涝年 表(表 1).

可以看出，全球降水量的旱涝年有非常明显的 年代际变化. 仅一个旱年出现在 20 世纪 60 年代, 其 他都在 1972 年以后. 所有的涝年, 除 2000 和 1988 年以外, 都出现在 1975 年以前. 20 世纪 50 年代集中 了许多涝年.

\section{4 纬圈平均年降水量的长期变化}

模糊聚类分析方法结果表明，36 个纬圈年平均 降水量合并成 6 个纬度带是合适的(聚类图略). 从 表 2 看出, 除了北半球高纬度 $\left(60^{\circ} \sim 90^{\circ} \mathrm{N}\right)$ 降水略微 增加以外, 各纬带的降水都在减少. 北半球中、低纬 降水减少最明显, 趋势系数为 -0.35 , 其次是热带地 区. 但是因为热带地区降水量的平均值与方差最大, 所以降水量减少最多的地区是热带, 平均降水减少 为 $0.98 \mathrm{~mm} / \mathrm{a}$; 其次是 $20^{\circ} \sim 35^{\circ} \mathrm{N}$, 为 $0.61 \mathrm{~mm} / \mathrm{a}$, 都 超过全球平均的 $0.54 \mathrm{~mm} / \mathrm{a}$.

\section{5 全球陆地年降水场与 ENSO}

我们使用了 53 年资料, 所以有了足够的 ENSO 事件. 在 1948 2000 年中, 暖事件年有 14 年, 冷暖事 件年有 10 年.

暖事件年为: $1951,1953,1957,1963,1965,1968$, $1972,1976,1982,1986,1991,1993,1994$ 和 1997; 冷 事件年为: $1949,1954,1955,1964,1970,1973,1975$, 1984, 1988 和 1998.

表 1 1948 2000 年全球旱涝年(黑体为明显旱涝年)

\begin{tabular}{|c|c|c|c|c|c|c|c|c|c|c|c|c|c|c|}
\hline 全球涝年 & 1973 & 1988 & 1955 & 1956 & 1950 & 1954 & 2000 & 1964 & 1974 & 1961 & 1975 & 1949 & 1962 & 1960 \\
\hline 全球旱年 & 1991 & 1992 & 1993 & 1994 & 1982 & 1972 & 1986 & 1995 & 1985 & 1997 & 1965 & 1979 & 1976 & \\
\hline
\end{tabular}

表 26 纬带的格点数、年降水量的平均值与均方差 $($ 单位: $\mathrm{mm}$ )及趋势系数和回归系数 $(\mathrm{mm} / \mathrm{a})$

\begin{tabular}{lcccccc}
\hline & $\begin{array}{c}\text { 南半球高纬度 } \\
\left(90^{\circ} \sim 60^{\circ} \mathrm{S}\right)\end{array}$ & $\begin{array}{c}\text { 南半球中纬度 } \\
\left(60^{\circ} \sim 35^{\circ} \mathrm{S}\right)\end{array}$ & $\begin{array}{c}\text { 热带地区 } \\
\left(35^{\circ} \mathrm{S} \sim 20^{\circ} \mathrm{N}\right)\end{array}$ & $\begin{array}{c}\text { 北半球中、低纬 } \\
\left(20^{\circ} \sim 35^{\circ} \mathrm{N}\right)\end{array}$ & $\begin{array}{c}\text { 北半球中、高纬 } \\
\left(35^{\circ} \sim 60^{\circ} \mathrm{N}\right)\end{array}$ & $\begin{array}{c}\text { 北半球高纬度 } \\
\left(60^{\circ} \sim 90^{\circ} \mathrm{N}\right)\end{array}$ \\
\hline 格点数 & 1110 & 90 & 998 & 415 & 907 & 870 \\
平均值 & 361.7 & 1053.1 & 1287.3 & 625.5 & 604.4 & 288.6 \\
均方差 & 56.1 & 35.0 & 49.4 & 26.6 & -0.9 & -0.13 .4 \\
趋势系数 & -0.14 & -0.20 & -0.31 & -0.61 & -0.18 & 0.15 \\
回归系数 & -0.53 & -0.46 & -0.98 & & 0.10 \\
\hline
\end{tabular}




\section{1 陆地年大尺度降水场与 ENSO}

图 3(a) 是第一经验正交函数 (EOF) 的时间系数 (PC1)的直方图. 图中深黑色是暖事件年, 而灰色代表 冷事件年. 由图 3(a)看出, 在 14 个暖事件年中有 13 年 时间系数是负值. 相反, 10 个冷事件年的时间系数全 部是正值. 另外, 在所有的正的时间系数年中(共 27 年), 有 10 年是冷事件年, 仅 1 年(1953 年)是暖事件 年; 在最大的 10 个正时间系数年中, 有 8 年是冷事件 年, 没有暖事件年. 而在所有负的时间系数年中(共 26 年), 有 13 年是暖事件年, 没有冷事件年. 在最大的 9 个负时间系数年中, 有 7 年是暖事件年, 没有冷事件 年. 这些特征清楚地表明, 全球大尺度降水量场与 ENSO 关系密切. 因为, 大的正(负)时间系数对应全球 的涝(旱), 所以, 全球的旱涝与 ENSO 有关(见表 2).

\section{2 年平均降水量距平与 ENSO}

图 3(b)是全球陆地平均的年降水距平的直方图,
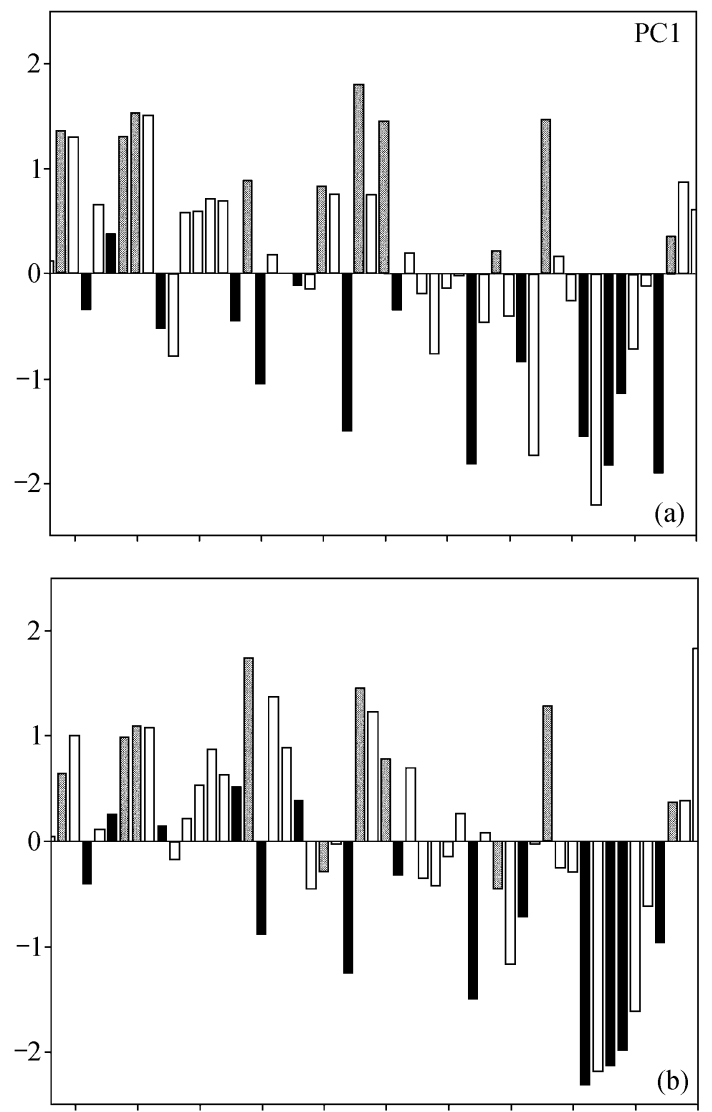

19501955196019651970197519801985199019952000 (年)

图 3 全球陆地年降水量第一 EOF 的时间系数(a)及全球陆 地平均年降水距平(b)的直方图(数值已标准化) 黑色代表暖事件年, 而灰色代表冷事件年
直接反映全球降水平均距平, 所以, 它的意义更清楚. 表 3 是全球陆地平均的年降水距平在冷暖事件年的 平均值及差值的 $t$ 检验, 可以看出, 暖事件年时, 全 球陆地平均的年降水量减少 $15.4 \mathrm{~mm}$, 而在冷事件年, 全球陆地平均的年降水量增加 $14.4 \mathrm{~mm}$. 这样, 暖事件 年要比冷事件年降水量减少 $29.8 \mathrm{~mm}$. 这个差值达到 0.01 的信度, 表明, 冷暖事件影响了全球年降水量.

表 3 全球陆地年平均的年降水距平与冷暖事件的关系

\begin{tabular}{lc}
\hline & 全球陆地平均的年降水距平 \\
\hline 平均值(暖事件年) & $15.4 \mathrm{~mm}$ \\
平均值(冷事件年) & $14.4 \mathrm{~mm}$ \\
差(暖事件年 -冷事件年) & $-29.8 \mathrm{~mm}$ \\
$t$ 统计量 & -4.38 \\
\hline
\end{tabular}

\section{3 纬带平均降水量与 ENSO}

计算了 6 纬带的年降水量在冷暖事件年的平均 值、差值及差值的 $t$ 检验. 结果表明, 6 个纬带的平均 的年降水量, 在暖事件年平均比冷事件年少. 但是, 在热带地区，暖事件年(冷事件年)的年降水量的平均 值分别是 $1248.0 \mathrm{~mm}(1332.3 \mathrm{~mm})$, 这种差异的 $t$ 统计 量为 -6.38 , 信度达到 0.0001 .

图 4 是 $35^{\circ} \mathrm{S} 20^{\circ} \mathrm{N}$ 年降水量的直方图, 图数值 已经标准化. 可以看出, 暖事件年的降水量比冷事件 年少. 从图 3 和 4 看出, 20 世纪 70 年代末开始, ENSO 发生了年代际变化, ENSO 的频率与强度都增 加了. 因为, ENSO 影响全球降水量, 所以, 20 世纪 70 年代末开始频繁发生的 ENSO 事件是全球年降水量 减少的一个重要的原因. 最近, 施能等人 ${ }^{1}$ 还比较详

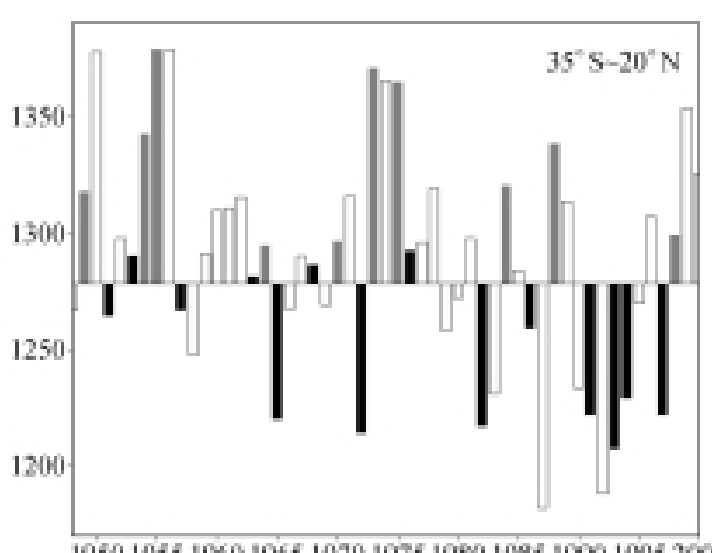

图 4 全球 $35^{\circ} \mathrm{S} 20^{\circ} \mathrm{N}$ 热带地区年降水量(单位: $\mathrm{mm}$ ) 的直方图

黑色代表暖事件年, 灰色代表冷事件年

1) Shi Neng, Chen Luwen, Xia Dongdong. A preliminary study on the globalland annual precipitation associated with ENSO during 1948 2000. Advance in Atmospheric Sciences (待发表) 
细地研究了 1948 2000 年 ENSO 事件与全球降水量 的关系.

\section{6 结论}

在 1948 2000 年期间，全球的年降水量有明显的 下降趋势, 并且在 1978 年左右发生突变. 平均每年 减少 $0.54 \mathrm{~mm}$, 并且除了高纬度 $\left(60^{\circ} \sim 90^{\circ} \mathrm{N}\right)$ 降水略 微增加以外, 各纬度带的平均降水量都表现为减 少的特征, 这个结果与 Hulme ${ }^{[5]}$ 的结果是不同的. Hulme 的结果是南半球的平均年降水量每年增加 1.4 $\mathrm{mm}$, 这是由于他只研究了 1951 1980 年的年降水量. 而 20 世纪 80 年代以后全球降水量的减少是明显的, 特别是 $35^{\circ} \mathrm{S} \sim 35^{\circ} \mathrm{N}$ 和 $35^{\circ} \mathrm{S} \sim 20^{\circ} \mathrm{N}\left(20^{\circ} \mathrm{N} \sim 35^{\circ} \mathrm{N}\right)$ 降 水分别减少 $0.98 \mathrm{~mm} / \mathrm{a}(0.61 \mathrm{~mm} / \mathrm{a})$. 我们还划分了 1948 2000 年的全球旱涝年, 指出全球旱涝年也有年 代际变化, 并研究了年降水量与 ENSO 的关系. 在暖 事件年全球陆地平均每年减少的降水量为 $15.4 \mathrm{~mm}$, 而在冷事件年, 年降水量每年增加 14.4 mm. 20 世纪 70 年代末开始频繁发生的 ENSO 事件是全球年降水 量减少的一个重要的原因. 本文仅是研究全球的大 尺度年降水量的结果. 降水量的时、空变率很大, 全 球季节的及区域的降水长期变化是需要今后进一步 研究的.

致谢本工作受国家自然科学基金(批准号: 40275028) 资助.

\section{参考文献}

1 Gruza G V, Apasova E G. Climatic variability in the monthly sums of precipitation in the northern hemisphere. Meteorol Gidrol, 1981, 5: $5 \sim 16$

2 Diaz H F, Bradley R S, Eischeid J K, et al. Precipitation fluctuation over global land areas since the late 1800's. J Geophs Res, 1989, 94(D1): 1195 1210

3 Bradley R S, Diaz H F, Eischeid J K, et al. Precipitation fluctuations over northern hemisphere land areas since the Mid-19th century. Science, 1987, 237: 171 175

4 Vinnikov K Y, Groisman P Y, Lugina K M. Empirical data on contemporary climate changes (temperature and precipitation). J Climate, 1990, 3: 662 677

5 Hulme M. A 1951-80 global land precipitation climatology for the evaluation of general circulation models. Climate Dynamics, 1992,
7: $57 \sim 72$

6 Dai A, Fung I Y, Genio A D D, et al. Surface observed global land precipitation variations during 1900 1988. J Climate, 1997, 10(11): 2943 2962

7 Hulme M. Estimation global changes in precipitation. Weather, 1995, (1): 34 42

8 Xie P, Arkin P A. Global precipitation: A 17-year monthly analysis based on gauge observations, satellite estimates, and numerical model outputs. Bulletin of the American Meteorological Society, 1997, 78(11): 2539 2558

9 Ropelewsi C F, Halpert M S. Global and regional scale precipitation patterns associated with Elnino/Oscillation. Mon Wea Rev, 1987, 115: 1606 1626

10 Lau K M, Sheu P J. Teleconnection in global rainfall, seasonal to inter-decadal time scales anomalies. In: Glantz M, Katz R, Nicholls N, eds. Teleconnections Linking Worldwide Climate Anomalies. Cambridge: Cambridge University Press, 1991. $227 \sim 256$

11 Rasmusson E M, Carpenter T H. Variation in tropical sea surface temperature and surface wind field associated with the Southern Oscillation/ Elnino. Mon Wea Rev, 1982, 110: 354 384

12 Bradley R S, Diaz H F, Kiladis G N, et al. ENSO signal in continental temperature and precipitation records. Nature, 1987, 327: 197 201

13 Wang B. Transition from a cold to a warm state of the Elniño-Southern Oscillation cycle. Met Atmos Phys, 1994, 48: $1 \sim 16$

14 Chen M P, Janowiak J E, Arkin P A. Global precipitation: a 50 year monthly analysis based on gauge observations. Journal of Hydrometeorology, 2002, 3(3): 249 266

15 Shi Neng, Deng Ziwang. Space/time features of the secular trend variation in 1951 1998 Northern 500hPa height. Meteorolo Atmos Phys, 2000, 73: 35 46

16 Shi Neng. Secular variation of winter atmospheric teleconnection pattern in the Northern hemisphere and its relation with China's climate change. Acta Meteorologica Sinica, 1996, 54(6): 676 683

17 Livezey R E, Chen W Y. Statistical field significance and its determination by Monte Carlo techniques. Mon Wea Rev, 1983, 111(1): 46 59

(2002-05-27 收稿，2002-07-30 收修改稿) 